\title{
Quantum fluctuations in the mazer
}

\author{
Jonas Larson \\ NORDITA, 10691 Stockholm, Sweden \\ E-mail: jolarson@kth.se
}

\begin{abstract}
.
Quantum fluctuations in the mazer are considered, arising either from the atomic motion or from the quantized intracavity field. Analytical results, for both the meza and the hyperbolic secant mode profile, predict for example an attenuation of tunneling resonances due to such fluctuations. The case of a Gaussian mode profile is studied numerically using a wave packet propagation approach. The method automatically takes into account fluctuations in the atomic motion and the dynamics is especially considered at or adjacent to a tunnel resonance. We find that the system evolution is greatly sensitive to the atom-field detuning, bringing about a discussion about the concept of adiabaticity in this model. Further, a novel collapse-revival phenomena is demonstrated, originating from the quantum fluctuations in the atomic motion rather from field fluctuations as is normally the case.
\end{abstract}

PACS numbers: 42.50.Pq, 03.65.Xp, 32.80.Qk, 03.75.-b

\section{Introduction}

In recent years, the field of experimental cavity quantum electrodynamics (QED) has expanded beyond the setup of single thermal two-level atoms traversing the cavity one by one [1, 2, 3. Cavity QED now includes the realization of solid state q-dots coupled to cavity fields [4, trapped ion-cavity systems [5], Bose-Einsten condensates coherently interacting with single cavity modes [6] and ultracold atomic many-body systems inside cavities; e.g., bistability behavior [7, cavity cooling [8] and self-organization of atoms in regular patterns [9]. Reaching these new regimes of cavity QED has been possible due to the progress in the cooling, trapping and coherent control of atoms [10. Actually, atomic velocities of the order of $1 \mathrm{~cm} / \mathrm{s}$ or even slower are achievable [11. This opens up the possibility to experimentally demonastrate the mazer (Microwave Amplification via $z$-motion-induced Emission of Radiation), where ultracold, rather than thermal, two-level atoms are let to pass the cavity [12, 13].

In the ultracold regime, the kinetic energy of the atom is of the order of the atom-field interaction energy and must be treated quantum mechanically. Early on, it was predicted that atoms can be back scattered by the high- $Q$ cavity vacuum induced potential [12] or even trapped by the intracavity field [14, which indeed was later experimentally obtained for single photon fields [15]. Later, in a series of papers by the late Walther and coworkers, it was shown that in this new regime the emission probability for an excited atom traversing the cavity is greatly affected by the quantized atomic motion $[13$. For certain resonance conditions, where the atomic de Broglie wavelength match resonantly with the cavity length, the atom tunneling and emission probabilities are greatly increased. Such resonances lead to new phenomena of the field statistics in the micromaser pumped by excited atoms. Following [13, a set of papers where published on the mazer. Most of which consider analytically solvable mazer models, namely the atom-field detuning vanishes and the cavity mode profile has the shape of a meza function or a hyperbolic secant [16, 17, 18, 19, 20. These all assume the zero detuning situation where the mazer problem can be decoupled into two separate 1-D scattering problems, while for a non-zero detuning the dynamics is indeed more complex and the full coupled system must be considered. This was the subject of 
[21, where the problem of a non-zero detuning in the case of a meza function coupling was solved analytically (see also 22 ). Further, the situation of a sinusoidal mode profile with one or a few wavelengths and a Gaussian shape have been analyzed by means of approximate or numerical methods in [23] and [18] respectively. The limit in which the mode profile is "delta"-shaped has as well been discussed 24, 25. The mazer problem for a sinusoidal coupling with several wavelengths was investigated in [26] and the model was found to exhibit very different characteristics due to the quasi-periodicity, see also [27]. Extensions of the regular mazer setup have been considered in numerous works, e.g. a driven system [28, multi-level atoms and multi-photon processes [29, multi-cavity setups [16, 22, 30, Kerr and gravity effects on the mazer action [31, 32. Also semi-classical approaches have been studied, where for example the atomic motion is replaced by an effective time-dependence [33, 34.

None of the above references take into account fluctuations in the motion of the atom. In the standard atom cavity QED experiment in which the atom traverses the cavity, its position is fairly well localized in space, typically for a microwave setup within the order of $1 \mathrm{~mm}$ (where the mode waist $L$ is about 5-6 mm) [1]. Thus, in real experiments is the state of the atom given by a well localized wave packet rather than a traveling wave. Naturally, an uncertainty in position results in fluctuations in momentum which, on the other hand, affects the particle scattering. In this paper we address these issues both by generalizing known analytical models but also via numerical analysis of a Gaussian mode profile. The Gaussian coupling approximates the mode profile of a Fabry-Perot cavity which is usually used in micromaser experiments [1, 2]. A crucial feature of the Gaussian, as well for the hyperbolic secant, coupling is that they are smooth with a well defined analytical limit, contrary to the meza function. This give rise to qualitative different results compared with the meza case. Here we focus on these consequences and discuss the concept of adiabaticity in the mazer. One essential parameter for adiabaticity is the atom-field detuning and how it affects the dynamics is investigated. It is found that it plays a central role for the evolution. So far, non-zero detuning has only been considered for meza-mode couplings, which do not possess an adiabatic limit due to their discontinuities. Quantum fluctuations lead in general to a smearing out of physical observables, but we demonstrate that it can also render new collapse-revival structures.

The general mazer concept and the model system are introduced in the section 2 . The various reflection and transmission coefficients, as well as appropriate bases, are given. Our analytical results are presented in section 3. where quantum fluctuations in both the meza and hyperbolic secant cases are considered. The following section 4 is devoted to numerical research of the Gaussian mode profile for zero and non-zero detunings. We apply a split-operator approach that invitably takes into account fluctuations in the motion of the atom. The effect of the detuning parameter is studied as well as the system dynamics during the scattering process. In the final section 5 we give a summery and discuss experimental prospects.

\section{The model system}

We consider a two-level atom with internal states $|g\rangle$ and $|e\rangle$ and center-of-mass (c.o.m.) momentum and position $\hat{p}$ and $\hat{z}$, which dipole interacts with a single quantized mode of a cavity. The effective Hamiltonian in the rotating wave approximation reads

$$
\tilde{H}=\frac{\hat{p}^{2}}{2 m}+\hbar \omega\left(\hat{a}^{\dagger} \hat{a}+\frac{1}{2}\right)+\frac{\hbar \Omega}{2} \hat{\sigma}_{z}+\hbar \lambda(\hat{z})\left(\hat{a}^{\dagger} \hat{\sigma}^{-}+\hat{\sigma}^{+} \hat{a}\right)
$$

where $m$ is the mass of the atom, $\omega$ the field frequency, $\Omega$ is the atomic transition frequency and $\lambda(\hat{z})$ is the effective position dependent atom-field coupling. Here $\hat{a}$ and $\hat{a}^{\dagger}$ are the annihilation and creation operators for the field and the $\sigma$-operators are the regular Pauli matrices, $\hat{\sigma}_{z}=|e\rangle\langle e|-| g\rangle\left\langle g\left|, \hat{\sigma}^{-}=\right| g\right\rangle\langle e|$ and $\hat{\sigma}^{+}=|e\rangle\langle g|$. The number of excitations is conserved and it may be used to define an interaction picture rotating with $\omega$. This introduces the atom-field detuning $\Delta=\Omega-\omega$ and the transformed Hamiltonian becomes (we let $\hbar=m=1$ )

$$
H=\frac{\hat{p}^{2}}{2}+\frac{\Delta}{2} \hat{\sigma}_{z}+\lambda(\hat{z})\left(\hat{a}^{\dagger} \hat{\sigma}^{-}+\hat{\sigma}^{+} \hat{a}\right) .
$$


Due to this symmetry can the Hamiltonian be written on $2 \times 2$ block diagonal form within its internal states. For a given number of excitations, corresponding to one of the $2 \times 2$-blocks, it is often convenient to turn to a dressed representation by transforming $H$ with [35]

$$
U=\left[\begin{array}{cc}
\cos (\theta) & \sin (\theta) \\
-\sin (\theta) & \cos (\theta)
\end{array}\right]
$$

where $\tan (2 \theta)=2 \lambda(\hat{z}) \sqrt{n} / \Delta$. This defines the dressed internal states

$$
\begin{aligned}
& \left|\gamma_{n}^{+}\right\rangle=\cos (\theta)|n, e\rangle+\sin (\theta)|n+1, g\rangle, \\
& \left|\gamma_{n}^{-}\right\rangle=-\sin (\theta)|n, e\rangle+\cos (\theta)|n+1, g\rangle .
\end{aligned}
$$

Here, $|n, e\rangle$ and $|n+1, g\rangle$ are the states with $n$ photons and the atom excited or $n+1$ photons and the atom in its ground state respectively. These states are referred to as bare internal states. The bare state $|0, g\rangle$ is decoupled from any other states, which, however is not true beyond the rotating wave approximation. We further have that $U$ diagonalizes the last two terms in the Hamiltonian (2) whose eigenvalues read

$$
V_{n}^{ \pm}(z)= \pm \sqrt{\left(\frac{\Delta}{2}\right)^{2}+\lambda^{2}(z)(n+1)}, \quad n=0,1,2, \ldots .
$$

We call these adiabatic potentials whenever $\Delta \neq 0$. Since the operator $U$ is $\hat{z}$-dependent it will not commute with the first term of $H$, causing non-diagonal terms which couples the two dressed internal states [35]. However, in the limiting cases $\Delta \rightarrow \pm \infty, 0$ one has that these non-adiabatic couplings vanish. Thus, for $\Delta=0$ we may decouple the two equations and we note that in this special case $\cos (\theta)=\sin (\theta)=1 / \sqrt{2}$. We distinguish this limit with the ones of large detuning (adiabatic limit) and call it diabatic limit [36].

Due to the coupled two-level structure, the dynamics may become rather complicated. An atom entering the interaction region will in general feel both an attractive and a repulsive potential. Despite the coupled dynamics, transmission and reflection coefficients for the two internal states can be well defined. In particular, for the dressed basis we call these $\rho_{n}^{ \pm}$and $\tau_{n}^{ \pm}$. It follows that the coefficients in the bare basis are related to the dressed ones via [13]

$$
\begin{array}{rlrl}
R_{e n} & =\frac{1}{2}\left(\rho_{n}^{+}+\rho_{n}^{-}\right), & R_{g n}=\frac{1}{2}\left(\rho_{n}^{+}-\rho_{n}^{-}\right), \\
T_{\text {en }}=\frac{1}{2}\left(\tau_{n}^{+}+\tau_{n}^{-}\right), & T_{g n}=\frac{1}{2}\left(\tau_{n}^{+}-\tau_{n}^{-}\right) .
\end{array}
$$

The $R$-coefficients squared give the probability for reflection of the atom in state $e$ or $g$ respectively and correspondingly $T$ gives the transmission. Thus, we have the transmission probability, given an excitation $n$,

$$
P_{\text {trans }}^{n}=\left|T_{e n}\right|^{2}+\left|T_{g n}\right|^{2}
$$

and similarly for the reflection. It should be pointed out that the mazer scattering process is different from the regular 1-D ones often presented in textbooks [37. This comes about because of the coupled dynamics between the internal states, and in particular, in some limiting cases can the particle be said to be scattered simultaneously to an attractive and repulsive potential. This, in fact, leads to new phenomena [16, not encountered in the standard situations. Another property of interest is the von Neumann entropy which gives a measure of entanglement shared between the field and the atom. Defining the reduced density operator for the field as $\rho_{f}=\operatorname{Tr}_{a t}[\rho]$, where $\rho$ is the full system density operator and the trace is over atomic degrees of freedom, one has the von Neumann entropy

$$
S_{n}=-\operatorname{Tr}\left[\rho_{f} \log \left(\rho_{f}\right)\right] .
$$


The field and atomic entropies are identical for initial pure states [38. In terms of the coefficients (6) it follows that

$$
\begin{aligned}
S_{n}= & -\left(\left|R_{e n}\right|^{2}+\left|T_{e n}\right|^{2}\right) \log \left[\left(\left|R_{e n}\right|^{2}+\left|T_{e n}\right|^{2}\right)\right] \\
& -\left(\left|R_{g n}\right|^{2}+\left|T_{g n}\right|^{2}\right) \log \left[\left(\left|R_{g n}\right|^{2}+\left|T_{g n}\right|^{2}\right)\right] .
\end{aligned}
$$

Let us choose the initial state to be the uncorrelated state with the field in vacuum, the atom in its internal excited state and with some spatial wave function $\psi(z)$,

$$
\langle z \mid \Psi(t=0)\rangle=\psi_{i}(z)|0, e\rangle .
$$

Using the position-momentum relation

$$
\psi(z)=\int d k \tilde{\psi}(k) \mathrm{e}^{i k z}
$$

we can write down the wave function for large times, when the atom is far from the interaction regime, as [13]

$$
\begin{aligned}
\langle z \mid \Psi(t)\rangle= & \int d k \tilde{\psi}(k) \mathrm{e}^{-i\left(k^{2} / 2\right) t}\left\{\left[R_{e 0}(k) \mathrm{e}^{-i k z}+T_{g 0}(k) \mathrm{e}^{i k z}\right]|0 e\rangle\right. \\
& \left.+\left[R_{g 0}(k) \mathrm{e}^{-i k z}+T_{g 0}(k) \mathrm{e}^{i k z}\right]|1, g\rangle\right\} .
\end{aligned}
$$

Taken into account for fluctuations in momentum we define the total reflection and transmission coefficients

$$
\begin{aligned}
\left|\tilde{R}_{i 0}\right|^{2}=\int d k\left|\tilde{\psi}(k) R_{i 0}(k)\right|^{2}, & i=e, g, \\
\left|\tilde{T}_{i 0}\right|^{2}=\int d k\left|\tilde{\psi}(k) T_{i 0}(k)\right|^{2}, & i=e, g .
\end{aligned}
$$

With these, the transmission probability or the von Neumann entropy are readily modified. Noteworthy is that the coefficients (13) is as well easily generalized for general initial cavity fields, $|\phi\rangle=\sum_{n} c_{n}|n\rangle$ by weighting the corresponding coefficients by $\left|c_{n}\right|^{2}$ and summing over $n$.

\section{Analytical consideration}

Some of the outcomes from quantum fluctuations, both in the atomic c.o.m. motional state and in the cavity photon field, are demonstrated in this section using analytically solvable models. The sequential section continues the analysis by a numerical study of a more physically realistic situation. In both models considered here is the detuning $\Delta$ is assumed zero. In other words, the internal structure can be separated into two decoupled equations. The atomic motional wave function is taken to be a Gaussian, centered at $k_{0}$ and with width $\Delta_{k}$,

$$
\tilde{\psi}(k)=\left(\frac{1}{\pi \Delta_{k}^{2}}\right)^{1 / 4} \mathrm{e}^{-\frac{\left(k-k_{0}\right)^{2}}{4 \Delta_{k}^{2}}} .
$$

If not mentioned, the cavity field is initially in the vacuum.

\subsection{Meza function}

The simplest solvable model, and also most extensively studied, assumes a constant atom-field coupling within and zero outside the cavity region [12, 13, 16, 17.

$$
\lambda(z)= \begin{cases}\lambda_{0} & \text { for } 0<z<l \\ 0 & \text { elsewhere }\end{cases}
$$



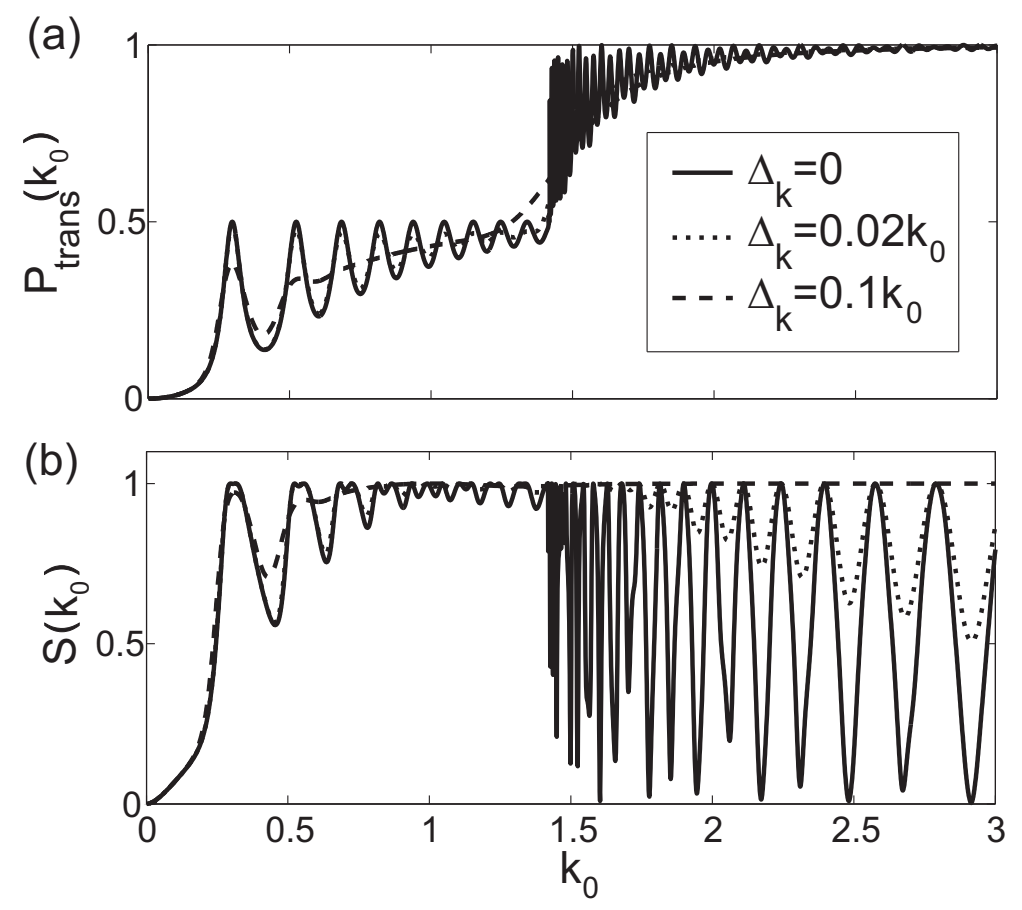

Figure 1. The transmission probability $P_{\text {trans }}$ and the field entropy $S$ (for the meza function) as function of atomic c.o.m. momentum $k_{0}$. Solid lines correspond to the results (7) and (9), while dashed and dotted lines take into account for momentum fluctuations. Here $\lambda_{0}=1$ and $L=50$.

The dressed transmission and reflection coefficients read [13]

$$
\begin{gathered}
\rho_{n}^{ \pm}=i \Delta_{n}^{ \pm} \sin \left(k_{n}^{ \pm} L\right) \tau_{n}^{ \pm}, \\
\tau_{n}^{ \pm}=\exp (-i k L)\left[\cos \left(k_{n}^{ \pm} L\right)-i \Sigma_{n}^{ \pm} \sin \left(k_{n}^{ \pm} L\right)\right]^{-1},
\end{gathered}
$$

where

$$
\begin{gathered}
k_{n}^{ \pm}=\sqrt{k^{2} \mp \kappa_{n}^{2}}, \\
\Delta_{n}^{ \pm}=\frac{1}{2}\left(\frac{k_{n}^{ \pm}}{k}-\frac{k}{k_{n}^{ \pm}}\right), \\
\Sigma_{n}^{ \pm}=\frac{1}{2}\left(\frac{k_{n}^{ \pm}}{k}+\frac{k}{k_{n}^{ \pm}}\right), \\
\kappa=\sqrt{2 \lambda_{0}}, \\
\kappa_{n}=\kappa \sqrt[4]{n+1} .
\end{gathered}
$$

The discontinuity of the atom-field coupling, once the problem is presented in the dressed basis, will render delta-like non-adiabatic coupling terms [35]. This singularity naturally affects the system properties and in particular are such effects believed to be absent in realistic experimental models to be discussed in the next Section.

The results for the transmission probability and the von Neumann entropy are presented in Figures 1 and 2. The first figure shows how the quantities depend on the atomic c.o.m. momentum $k_{0}$. The solid lines 

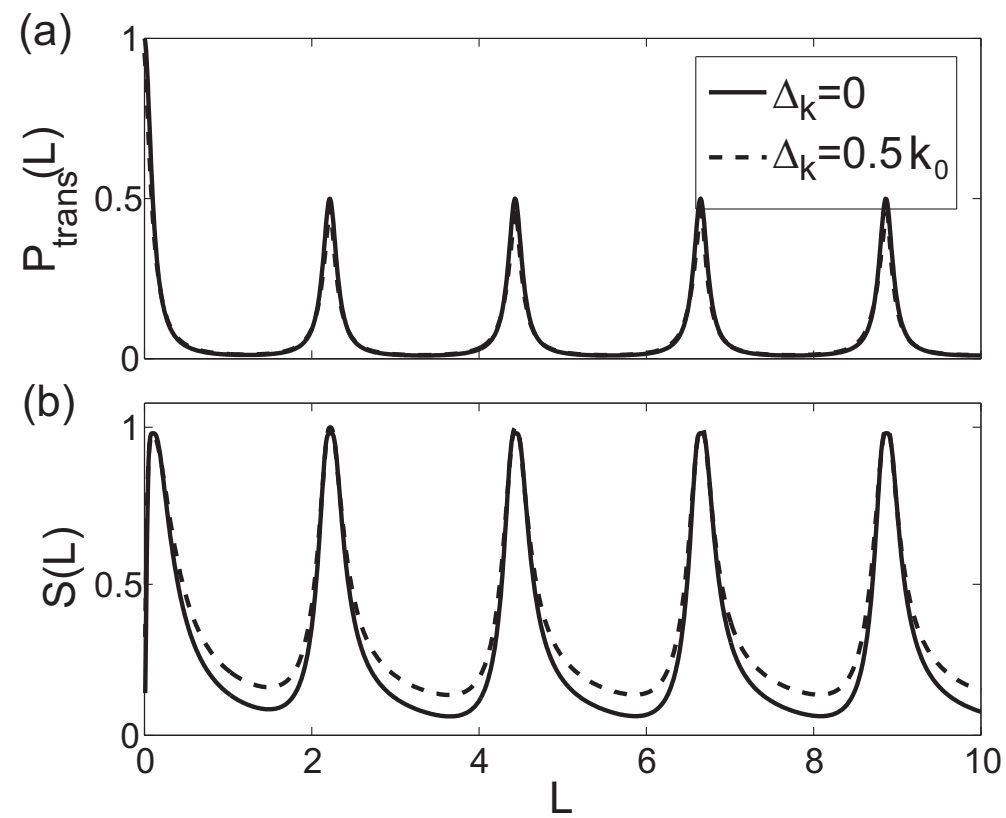

Figure 2. The transmission probability $P_{\text {trans }}$ and the field entropy $S$ (for the meza function) as function of mode length $L$. As in Figure 10 solid lines display the results (7) and (9), while the others include momentum fluctuations. Here $\lambda_{0}=1$ and $k_{0}=\lambda_{0} / 10$.

give the zero fluctuation case (7) and (9), the dotted lines are calculated with a momentum fluctuation of 2 $\%$ of the c.o.m. momentum, $\Delta_{k}=0.02 k_{0}$, and for the dashed line $\Delta_{k}=0.1 k_{0}$. It is clear how the quantum fluctuations smear out any variations of the two quantities, including the tunneling resonances in the mazer regime $0<k_{0}<\sqrt{2}$. The atom-field coupling is here $\lambda_{0}=1$ such that for momentum $k_{0}>\kappa_{0}=\sqrt{2 \lambda_{0}}$ is the atomic kinetic energy larger than the potential barrier and the atomic transmission is abruptly increased beyond $\kappa_{0}$. In this Rabi regime one notes how the atom-field Rabi oscillations begin to form. The period of Rabi oscillations decreases faster than the width $\Delta_{k}$ increases, resulting in that these oscillations are captured in the dotted line $\left(\Delta_{k}=0.02 k_{0}\right)$ for large $k$. The atom-field entanglement is maximized when the transition probability reaches $1 / 2$ in the mazer regime. In the Rabi regime, the entanglement follows the swapping of the excitation between the field and the atom. In Figure 2, we show the same quantities when instead the coupling length $L$ is varied. In this case, the results are insensitive to small momentum fluctuations and we therefore only show the results for $\Delta_{k}=0.5 k_{0}$. The resonances envisaged in the plots occur for $L=m \pi / \kappa_{n=0}$. Note that the fluctuations tend to increase the quantum correlations between the atom and the field.

How an uncertainty in the field statistics affects the transmission probability is depicted in Figure 3. The upper plot (a) shows the transmission probability for an initial coherent field state

$$
\left|c_{n}\right|^{2}=\frac{n_{0}^{n} \mathrm{e}^{-n_{0}}}{n !}
$$

with mean photon number $n_{0}=0, n_{0}=0.2$ and $n_{0}=1$. Figure $3(\mathrm{~b})$ gives the same but for an initial thermal field state

$$
\left|c_{n}\right|^{2}=\frac{n_{0}^{n}}{\left(n_{0}+1\right)^{n+1}} .
$$

It is found that the tunneling resonances are very sensitive to fluctuations in the cavity field. However, in the microwave regime of cavity QED, a thermal field with $n_{0}=1\left(n_{0}=0.2\right)$ correspond to a temperature of 


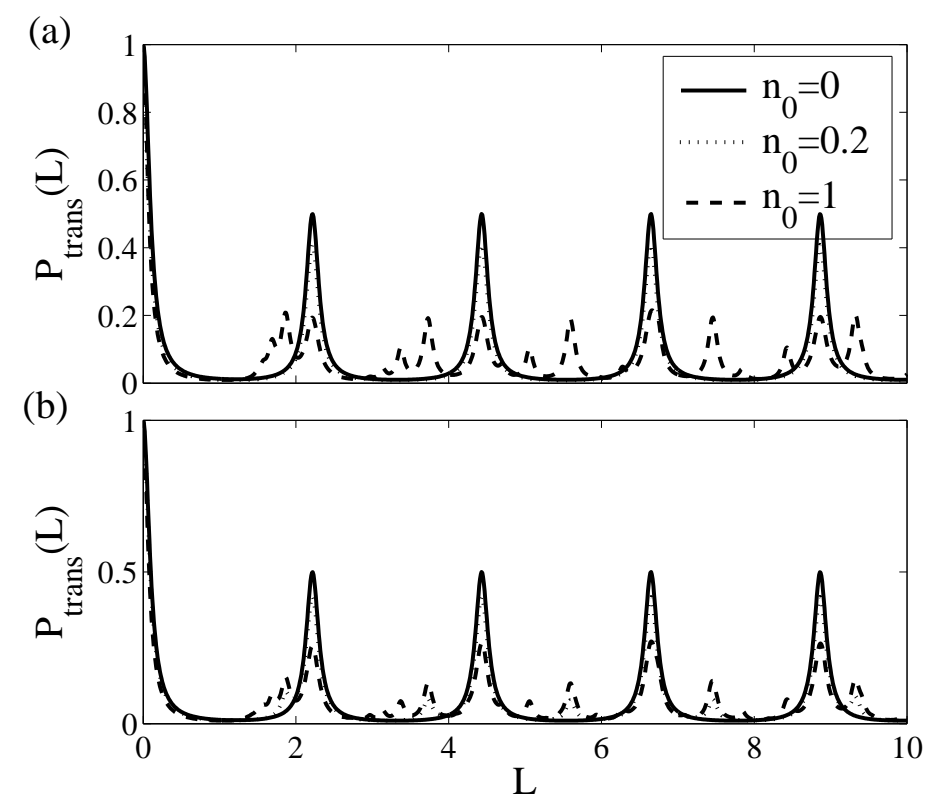

Figure 3. The transmission probability $P_{\text {trans }}$ (for the meza function) as function of mode length $L$ for an initial coherent cavity field (a) and an initial thermal cavity field (b). The average number of photons is $n_{0}=0$ (solid line), $n_{0}=0.2$ or $n_{0}=1$ (dashed line) and the other parameters are as in Figure 2

0.5 (0.2) degrees Kelvin, which is way above current effective experimental temperatures [1]. The secondary peaks showing up for non-zero $n_{0}$ are the tunneling resonances arising from other photon quantum numbers $n$ differing from $n=0$.

\subsection{Hyperbolic secant function}

To simulate a more realistic situation we consider a smooth atom-field coupling

$$
\lambda(z)=\lambda_{0} \operatorname{sech}^{2}\left(\frac{z}{L}\right)
$$

where $\lambda_{0}$ is the effective atom-field coupling and $L$ determines the mode waist length. The scattering problem analytically solvable in the special case of zero detuning $\Delta=0$, with dressed reflection and transmission coefficients [13]

$$
\begin{gathered}
\rho_{n}^{ \pm}(k)=\frac{\Gamma(i p L) \Gamma(1-i k L)}{\Gamma\left(1 / 2+i \xi_{n}^{ \pm}\right) \Gamma\left(1 / 2-i \xi_{n}^{ \pm}\right)} \tau_{n}^{ \pm}, \\
\tau_{n}^{ \pm}(k)=\frac{\Gamma\left[1 / 2-i\left(i k L+\xi_{n}^{ \pm}\right)\right] \Gamma\left[1 / 2-i\left(k L-\xi_{n}^{ \pm}\right)\right]}{\Gamma(-i k L) \Gamma(1-i k L)},
\end{gathered}
$$

where

$$
\xi_{n}^{ \pm}=\sqrt{ \pm 2 \lambda_{0} L^{2} \sqrt{n+1}-1 / 4}
$$

In terms of $\kappa_{n}$ (as defined above), the tunneling resonances are given by $\kappa_{n} L=\sqrt{m(m+1)}$ for $m=1,2,3, \ldots$ [13. The transmission probability $P_{\text {trans }}^{n}\left(k_{0}\right)$ and the von Neumann entropy $S\left(k_{0}\right)$ are displayed in Figure 4 as 

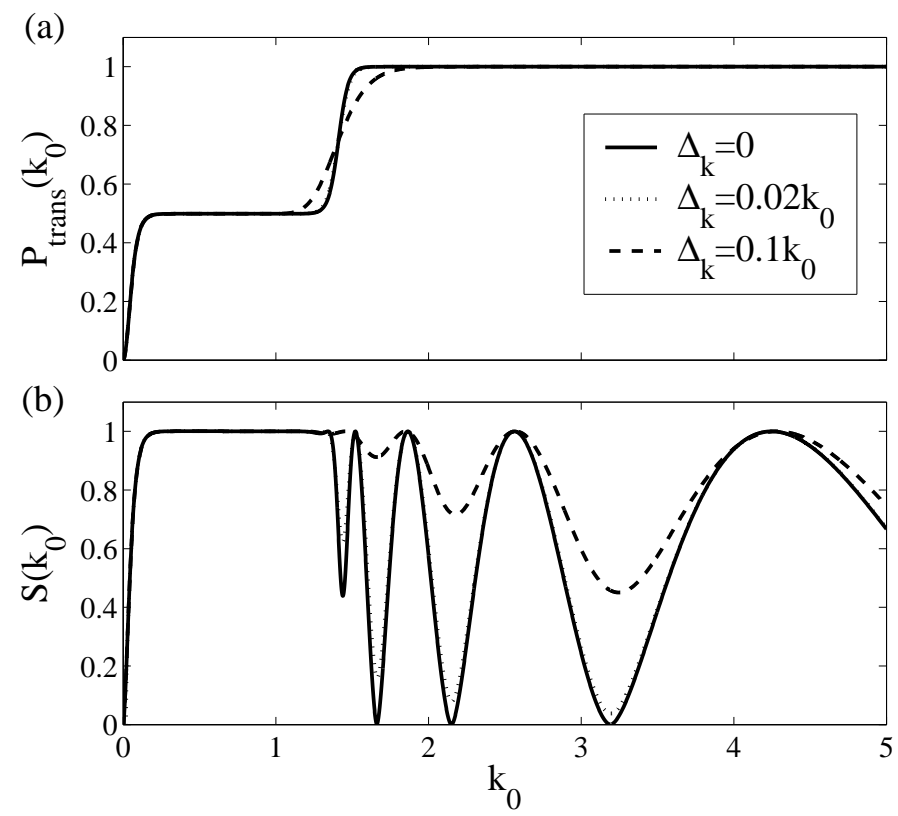

Figure 4. The transmission probability $P_{\text {trans }}$ and the field entropy $S$ (for the hyperbolic secant function) as function of the atomic c.o.m. momentum $k_{0}$. Contrary to the meza function, the transition probability equals $1 / 2$ and the the atom-field becomes maximally entangled for most of the momenta $k_{0}$ in the mazer regime $k_{0}<\lambda_{0} \sqrt{2}$. Here, as in Figure $1 \lambda_{0}=1$ and $L=5$.

function of $k_{0}$. The solid line gives the quantities for zero momentum fluctuations, while the dotted and dashed curves take into account for an uncertainty in the momentum distribution. It is seen that the transmission probability is constant in the mazer regime for this smooth coupling. In the Rabi regime is the transmission probability unity throughout composite to the meza function, which is an effect of the smoothness of the coupling. From the entanglement presented in plot (b) is it clear that in this regime the system Rabi oscillates. Comparing Figures 1 and 4, the effect of the non-analyticity of the meza function coupling is visible from the irregular behaviour of Figure 1. Again, the momentum fluctuations tend to blur the variations of the curves.

The dependence of the cavity mode waist $L$ is depicted in Figure 5 . As $L$ increases the transmission probability $P_{\text {trans }}(L)$ and von Neumann entropy $S(L)$ approaches $1 / 2$ and 1 respectively, which therefore deviate considerably from the results for the meza function presented in Figure 2. It is known that for large $L$, the adiabaticity is in general increased [35. Here, however, do we consider the case of $\Delta=0$ and we thus are in the strict diabatic limit rather than the adiabatic one.

\section{Numerical consideration}

In this section we consider a more realistic situation. The atoms perpendicularly traverse the field of a FabryPerot cavity, in which the mode shape is given by a Gaussian [1]

$$
\lambda(z)=\lambda_{0} \mathrm{e}^{-\frac{z^{2}}{L^{2}}} .
$$

The case of a Gaussian atom-field coupling in the mazer was considered numerically in [18, and the tunneling resonances where compared with the ones of the hyperbolic secant of the previous section. We, however, use a completely different numerical approach, namely a wave packet propagation procedure more commonly used 

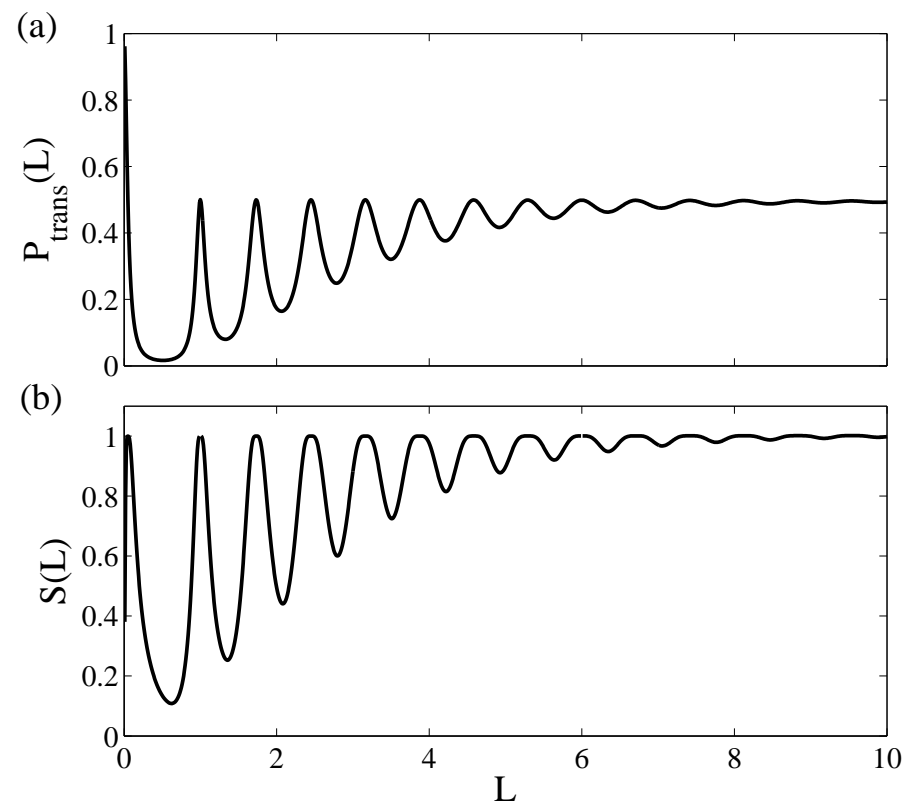

Figure 5. The transmission probability $P_{\text {trans }}$ and the field entropy $S$ (for the hyperbolic secant function) as function of mode waist $L$. Contrary to the meza function, the tunneling resonances vanish for large $L$ where the transition probability approaches $1 / 2$ and the the atom-field system becomes maximally entangled, $S=1$. Here, as in Figure 2 are $\lambda_{0}=1$ and $k_{0}=\lambda_{0} / 10$.

in molecular and chemical physics [39]. An initial wave packet, which in our case is taken to be a minimal uncertainty Gaussian

$$
\Psi\left(z, t_{i}\right)=\left(\frac{1}{\pi \Delta_{z}^{2}}\right) \mathrm{e}^{-\frac{\left(z-z_{0}\right)^{2}}{4 \Delta_{z}^{2}}}|e, 0\rangle,
$$

is propagated in time under the corresponding Hamiltonian (2) using the split operator method [40]. Thus, the wave packet is obtained at any instant of time and physical quantities are easily extracted from it. In particular, the transmission probability is calculated as

$$
P_{\text {trans }}=\lim _{t \rightarrow+\infty} \int_{0}^{\infty} d z|\Psi(z, t)|^{2},
$$

where the time limit is taken such that the result has asymptotically converged. An advantage of this approach is that it automatically takes into account for motional quantum fluctuations via the width $\Delta_{z}$ of the wave packet. Contrary to Reference [18, we will be interested in the effect of having a non-zero atom-field detuning $\Delta$ as well as the full system dynamics during the scattering process.

\subsection{Tunneling}

As already pointed out, the meza mode profile is expected to give an incorrect descripsion of realistic experimental situations. Firstly, it does not have the actual shape of typical cavity mode profiles, and secondly, it is a non-analytic function. Indeed, it is known that discontinuities cause non-adiabatic behavior and cannot correctly reproduce realistic cavity QED situations [34. More realistic results are instead expected to be obtained from the solvable hyperbolic secant model. However, this system is only solvable for a zero detuning 

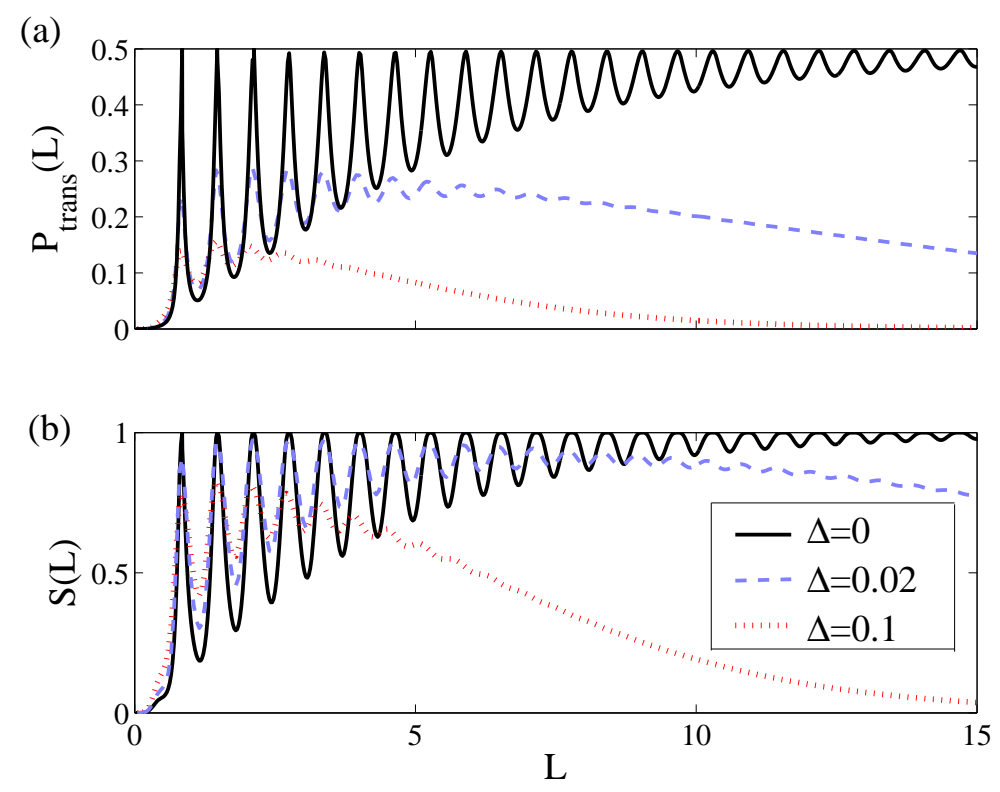

Figure 6. (Colour online) The transmission probability $P_{\text {trans }}$ and the von Neumann entropy $S$ (for the Gaussian mode profile) as function of mode waist $L$ and for three different detunings; $\Delta=0$ (solid line), $\Delta=0.02$ (dashed blue line) and $\Delta=0.1$ (dotted red line). Wide mode profiles convey a more adiabatic dynamics, causing a decrease in $P_{\text {trans }}$ and $S$. Here, $\Delta_{z}=15, \lambda_{0}=1$ and $k=\lambda_{0} / 10$.

21. Here we want to explore the scattering characteristics in the case of a Gaussian mode profile and compare with earlier results for the meza and hyperbolic secant.

In Figure [6 we present the numerical results for the transmission probability $P_{\text {trans }}(L)$ and the von Neumann entropy $S(L)$. The solid lines give the zero detuning case where the coupled equations can be decoupled into two scattering processes of attractive and repulsive potentials respectively. The tunneling resonances appears more often than for the hyperbolic secant coupling of Figure 5, which was also pointed out in [18. Another difference is the more rapid convergence to the asymptotic values $P_{\text {trans }}(L)=1 / 2$ and $S(L)=1$ for large $L$ of the hyperbolic secant profile compared to the Gaussian. This is a consequence of the longer tails of the hyperbolic secant and the smoother behaviour in general. This feature has been studied in a semi-classical approach for cavity QED models in [34, where it was found that the evolution is adiabatically more robust for a hyperbolic secant than for a Gaussian mode profile. However, it should be mentioned that the atomic c.o.m. motion was replaced by an effective time-dependence and the atomic kinetic energy exceeds the atom-field interaction energy in that work. The dotted and dashed lines in Figure 6 are the results obtained from having a non-zero detunings $\Delta$. Note that in both cases $\Delta<\lambda_{0}$ and still a great effect of the nonzero detuning is found. In other words, the tunneling dynamics is very sensitive to the parameter $\Delta$, and we especially note that this detuning dependence is clearly stronger for a Gaussian mode profile compared to a meza function 21. We discussed the adiabatic and diabatic potentials in Section 2 where $|\Delta| \rightarrow \infty$ gives the adiabatic limit. Figure 6 confirms our earlier conclusions that an increase in adiabaticity depletes the tunneling effect; large $\Delta$ and $L$ render an adiabatic evolution and in this regime the tunneling resonances are completely washed out. 


\subsection{Dynamics}

The atom-field dynamics in the mazer was briefly considered in 20 in terms of the characteristic tunneling time for a meza mode profile. Dynamics was also analyzed for an ultracold atom traversing a cavity with a cosine mode profile consisting of several periods 26. It was found that due to the quasi periodicity, an effective mass can be introduced in order to understand the evolution. The same system was investigated further in [25] studying the concept of a matter wave index of refraction.
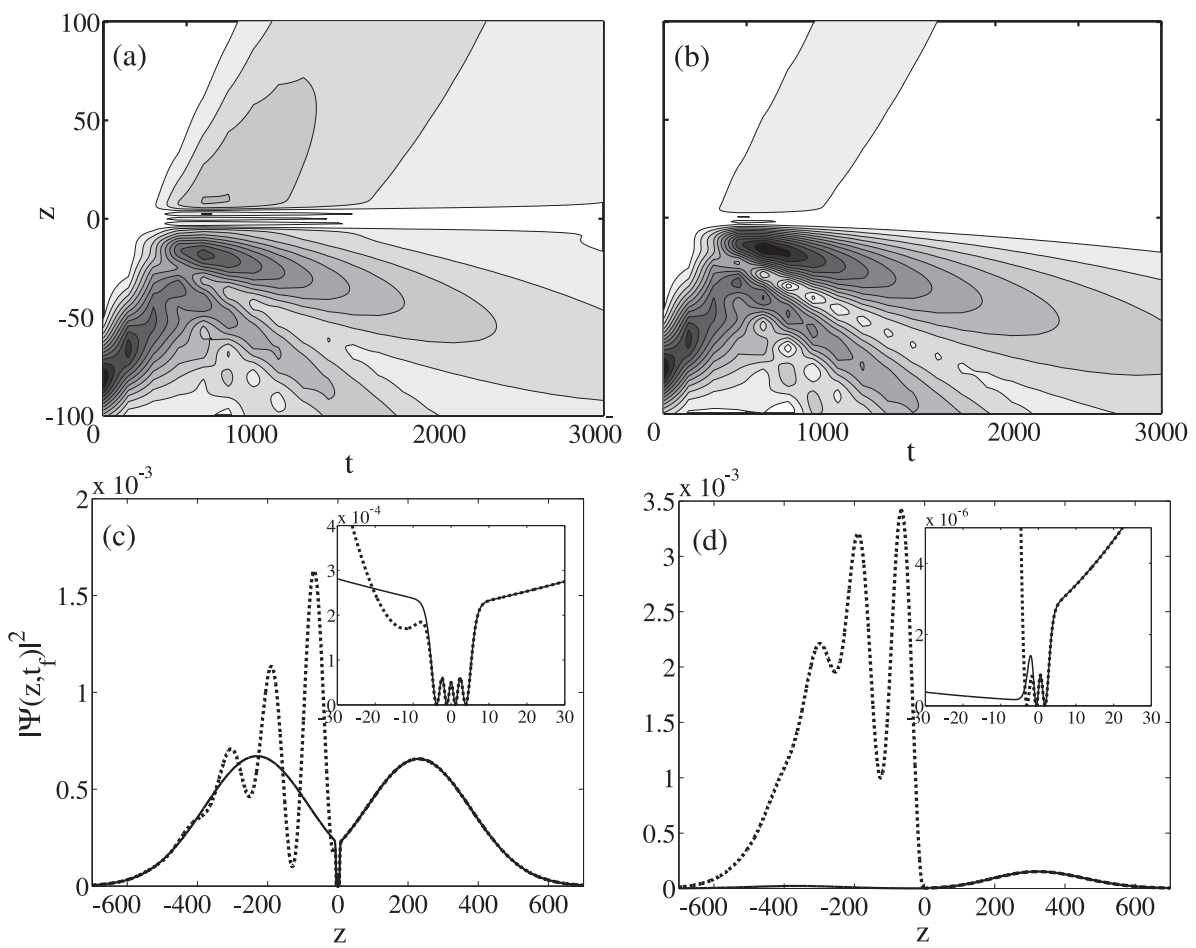

Figure 7. Time evolution of the probability distribution $\left|\psi_{e}(z, t)\right|^{2}+\left|\psi_{g}(z, t)\right|^{2}$ (a) and (b), and the individual amplitudes $\left|\psi_{e}(z, t=3000)\right|^{2}$ (dotted) and $\left|\psi_{g}(z, t=3000)\right|^{2}$ (solid) in (c) and (d) for a Gaussian mode profile. The left plots display the case where the parameters are chosen to give maximum tunneling (corresponding to the third resonance of Figure 6) while the right ones show the results with parameters in between two resonances (second minima of Figure 6). The insets zoom in on the wave packets in the classically forbidden region. Other parameters are as in Figure 6

We now turn to consider the Gaussian mode profile and the wave packet evolution while passing the interaction region, both when the tunneling condition is fulfilled and when it is not. We use the parameters of Figure 6 and chose $L=2.1245$ or $L=1.8108$ for the two situations respectively. These values of $L$ correspond to the third tunneling resonance and the second minima of Figure 6. We restrict the analysis to the zero detuning case. The results from the wave packet propagation are depicted in Figure 7, First, in (a) and (b) we give the total wave packet amplitude $|\Psi(z, t)|^{2}=\left|\psi_{e}(z, t)\right|^{2}+\left|\psi_{g}(z, t)\right|^{2}$, while (c) and (d) show the constituent amplitudes $\left|\psi_{e}(z, t=3000)\right|^{2}$ (dotted) and $\left|\psi_{g}(z, t=3000)\right|^{2}$ (solid) at time $t=3000$. The insets give a close-up of the amplitudes around the interaction region, $\lambda(z) \neq 0$. The difference between the two examples of being in or ouside the tunneling regime is evident from the plots. The typical decrease of the wave packet inside the classically forbidden regime throughout the evolution is also visible from the figure 41. The symmetric oscillations structure of the amplitude inside the forbidden region, seen in the inset of (c), is a characteristic of the tunneling resonance condition where the number of nodes depends on which resonance peak one considers; 
the number of atomic de Broglie wavelengths that fit the cavity length. Note that some oscillations are also present in the forbidden regime also in (d), but one should bare in mind the difference in amplitude (factor of 100) of the two insets.

\subsection{Motional induced collapse-revivals}

It is well known from quantum optics, and especially from cavity QED, that an uncertainty in the field state results in a collapse in the atom-field Rabi oscillations [42, 43]. The various photon number states that form the field state induce a series of different Rabi oscillations causing the collapse. At later times, the various terms comes back in phase, manifesting it selves in a revival. Also for the mazer has the collapse-revival effect been analyzed [44. In our model do we consider an initial vacuum and no collapse-revival pattern can arise due to field fluctuations. We will, however, show that the fluctuations in the atomic motional state may form a collapse-revival structure.
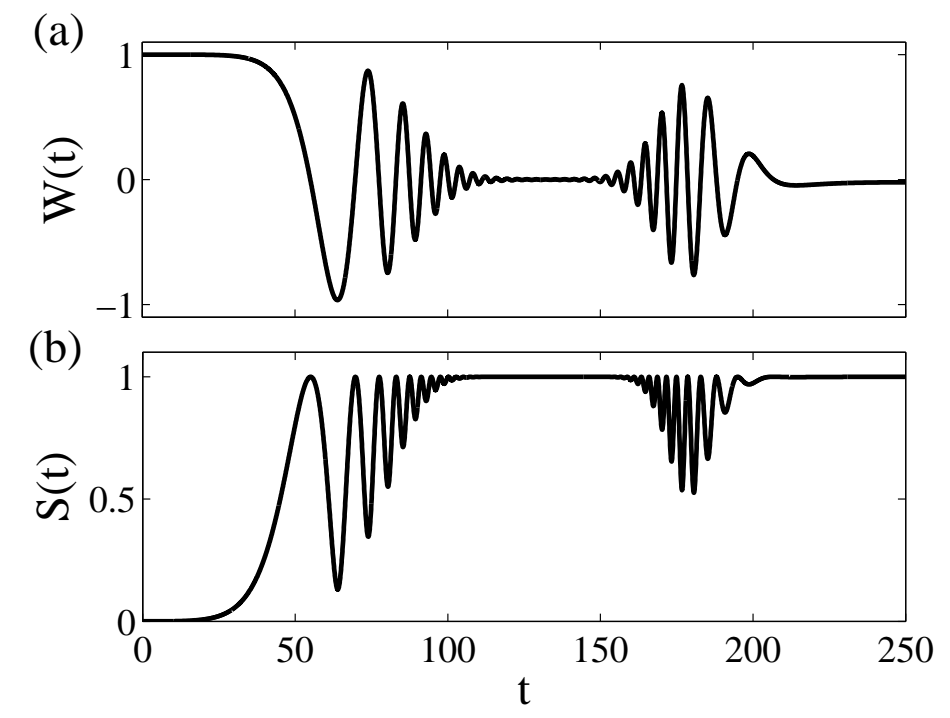

Figure 8. The atomic inversion $W(t)$ and the von Neumann entropy $S(t)$ (for the Gaussian mode profile) as function of time. Here $\Delta_{z}=10, \lambda_{0}=1 \Delta=0$ and $k_{0}=6$.

Assuming $k>\kappa_{n}$, but remaining in the regime where $k \sim \kappa_{n}$, the whole wave packet will be transmitted through the vacuum induced potential. From Figure 4 (b) we have that, while traversing the cavity field, the atom will Rabi oscillate with frequency $\lambda(z)$. However, since the coupling $\lambda(z)$ is not constant over the extent of the atomic wave packet, will the oscillation frequency vary within the packet. Entering the interaction region, the front of the wave packet sees a stronger amplitude of the atom-field coupling than the back of it. Letting $L / 2$ and $\tau=L / 2 k_{0}$ denote characteristic length and time scales respectively, and further assuming that the wave packet is to the left of the origin $z=0$ and that $\Delta_{z}<L$. Then, typically if $\left[\lambda(0)-\lambda\left(-\Delta_{z}\right)\right] \tau \sim \pi$ a collapse will occur in the dynamical quantities, such as the atomic inversion $W(t)=\left\langle\hat{\sigma}_{z}\right\rangle$ or the von Neumann entropy. After passing $z=0$, the coupling decreases and as the whole wave packet is to the right of the centre the situation will be the opposite; the front of the packet oscillates with a smaller frequency than the back. Thus, because of the symmetry of the Gaussian, the collapse will be reversed resulting in a revival, similar to time-reversal echo processes [43, 45]. The numerical results shown in Figure 8 for the inversion and the von Neumann entropy confirm the above arguments of a collapse-revival structer brought about due to momentum 
fluctuations. The imperfect revival is because wave packet spreading during the passage of the interaction region. Note that the revivals occurs due to the "time-reversal" like dynamics and not because of a discreteness in quantum numbers, as is the origin of regular revivals [42, 46].

\section{Conclusion}

In this work we have investigated the mazer beyond earlier studies by allowing for quantum fluctuations in the atomic motion and also in the field statistics. Known analytical models, the meza or the hyperbolic secant, have been extended to include such uncertainties and their effects have been discussed. Typically, the characteristics of various physical quantities, e.g. entanglement or transmission probability, are smeared out by these fluctuations. We have also shown that the fluctuations in general increase the amount of atom-field entanglement.

The more physically interesting situation, in terms of experimental realization, of a Gaussian mode profile was considered via a numerical wave packet approach. This method naturally takes into account for motional fluctuations and it is not limited to the situation of zero atom-field detuning. A consequence of a non-zero detuning is the fact that a decoupling of the internal two-level structure is not straightforward, apart from the limiting cases of adiabatic or diabatic evolution. We found that the system dynamics is very sensitive to the value of the detuning, which opened up questions about adiabaticity contra tunneling behaviour. Using our numerical propagation procedure we also studied the dynamics of the wave packet during the scattering against the vacuum potential. Finally we demonstrated how motional fluctuation can induce novel collapse-revival characteristics, different from the regular cavity QED collapse-revivals originating from fluctuations in the field statistics.

Experimentally, a difficulty with reaching the mazer regime is the extremely long characteristic time scales. The atoms are cooled down to the $\mathrm{nK}$ regime with typical c.o.m. velocity of $\mathrm{mm} / \mathrm{s}$ [13]. Using microwave cavities of mm-size 1 implies operational times of a few seconds. This exceeds typical atomic and cavity life-times which are of the order of ms. Another obstacle for these long processes is gravity, which however may be circumvented by using matter wave guides [4]. Atomic losses can be minimized by using a two-photon $R A M A N$ coupling, such that two meta stable atomic states are dispersively coupled via an excited state using one external laser field and the cavity field 48. This, however, introduces additional Stark shift terms in the Hamiltonian. To overcome the problem of cavity losses one could consider a pumped open cavity system. Another possibility is to try to speed up the process, for instance by considering a Bose-Einstein condensate coupled to the cavity [6], in which the effective atom-field coupling can be increased by a factor 1000 compared to the single atom case [49. In such a system, the intrinsic non-linearity leads to novel phenomena like bistability. This is under current investigation.

\section{Acknowledgments}

The author wishes to thank Profs. Stig Stenholm and Maciej Lewenstein for interesting discussions

\section{References}

[1] Brune M 2004 in Quantum Entanglement and Information Processing Eds. Esteve D, Raimond J.-M. and Dalibard J. (Amsterdam: Elsevier)

[2] Cavity Quantum Electrodynamics Ed. Bergman P R 1994 (London: Academic Press) Dutra S M Cavity Quantum Electrodynamics 2004 (Hoboken NY: Wiley-Interscience)

[3] Haroche S and Raimond J -M 2006 Exploring the Quantum (Oxford: Oxford University Press)

[4] Wallraff A, Schuster D I, Blais A, Frunzio L, Huang R S, Majer J, Kumar S, Girvin S M and Schoelkopf R J 2004 Nature 431 162

Press D, Gotzinger S, Reitzenstein S, Hofmann C, Löfler A, Kamp M, Forchel A and Yamamoto Y 2007 Phys. Rev. Lett. 98117402

Hennessy K, Badolato A, Winger M, Geraee D, Atature M, Gulde S, Fält S, Hu E L and Imamoglu A 2007 Nature 445896 
[5] Mundt A B, Kreuter A, Becher C, Leibfried D, Eschner J, Schmidt-Kaler F and Blatt R, 2002 Phys. Rev. Lett. 89103001 Keller M, Lange B, Hayasaka K, Lange W and Walther H 2004 Nature 4311075

[6] Slama S, Krentz G, Bux S, Zimmermann C and Courteille Ph W 2007 Phys. Rev. A 75063620 Brennecke F, Donner T, Ritter S, Bourdel T, Köhn M and Esslinger T 2007 Nature 450268 Truetlein P, Hunger D, Camerer S, Hänsch T W and Reichel J 2007 Phys. Rev. Lett. 99140403 Colombe Y, Steinmetz T, Dubois G, Linke F, Hunger D and Reichel J 2007 Nature 450272

[7] Gupta S, Moore K L, Murch K W and Stampern-Kurn D M 2007 Phys. Rev. Lett. 99213601

[8] Maunz P, Puppe T, Schuster I, Pinkse P W H, and Rempe G 2004 Nature 42850

[9] Black A T, Chan H W and Vuletć 2003 Phys. Rev. Lett. 91203001 Nagorny B, Elsässer and Hemmerich A 2003 Phys. Rev. Lett. 91153003 Kruse D, von Cube C, Zimmermann C and Courteille Ph W 2003 Phys. Rev. Lett. 91183601

[10] Meystre P 2001 Atom Optics (Berlin: Springer-Verlag) Metcalf H J and van der Straten P 2001 Laser cooling and trapping (Berlin: Springer-Verlag).

[11] Laurent Ph, Lemonde P, Simon E, Santarelli G, Clairon A, Dimarcq N, Petit P, Audoin C. and Salomon C 1998 Eur. Phys. J. D 3201

[12] Englert B G, Schwinger J, Barut A O and Scully M O 1991 Europhys. Lett. 1425

[13] Scully M O, Meyer G M and Walther H 1996 Phys. Rev. Lett. 764144 Mayer G M, Scully M. O. and Walther H 1997 Phys Rev. A 564142 Löffler M, Mayer G M, Schröder, Scully M O and Walther H 1997 Phys. Rev. A 564153 Schröder M, Vogel K, Schleich W P, Scully M O and Walther H 1997 Phys Rev. A 564164

[14] Haroche S 1991 Euro. Phys. Lett. 1419

[15] Ye J, Vernooy D W and Kimble H J 1999 Phys. Rev. Lett. 834987 Hood C J, Lynn T W, Doherty A C, Parkins A S and Kimble H J 2000 Science 2871447

[16] Agarwal G S and Arun R 2000 Phys. Rev. Lett. 845098

[17] Löffler M, Meyer G M and Walther H 1998 Europhyss Lett. 41593

[18] Bastin T and Solano E 2000 Comput. Phys. Commun. 124197

[19] Bastin T and Solano E 2003 Optics Commun. 217239

[20] Arun R and Agarwal G S 2002 Phys. Rev. A 64065802

[21] Bastin T and Martin J 2003 Phys. Rev. A 67053804 Martin J and Bastin T 2004 Euro. Phys. J. D 29133 Mahmoud A-A and Obada A-S F 2002 Mod. Phys. Lett. B 16117 Wang Q S, Du S D, Zhou L W, Chen X S and Bao L H 2004 Chinese Phys. Lett. 211749

[22] Seidal D and Muga J G 2007 Eur. Phys. J. D 4171

[23] Retamal J C, Solano E and Zugary N 1998 Opt. Commun. 15428

[24] Seidel D, Muga J G and Hegerfeldt G C 2006 J. Phys. B: At. Mol. Opt. Phys. 394673

[25] Larson J Ph.D. thesis: http:www.diva-portal.org/kth/theses/abstract.xsql?dbid=404

[26] Larson J, Salo J and Stenholm S 2006 Phys. Rev. A 72013814

[27] Saif F, Le Kien F and Zubairy M S 2001 Phys. Rev. A 64043812

[28] Xiong J and Zhang Z -M 2002 J. Phys. B 352159

[29] Zhang Z -M, Lu Z -Y and He L -S 1999 Phys. Rev. A 59808 Zhang Z -M and He L -S 1998 Opt. Commun. 15777 Arun R, Agarwall G S, Scully M O and Walther H 2000 Phys. Rev. A 62023809 Zhang Z -M, Xie S-W, Liang W Q and Zhou S -K 2000 J. Phys. B: At. Mol. Opt. Phys. 332125 Zhang Z -M, Chi S and Cheng B -C 2002 J. Opt. B 430 Arun R and Agarwal G S 2002 Phys. Rev. A 66043812 Obada A-S F and Mahmoud A-A 2003 International J. Mod. Phys. B 172735

[30] Zhang Z -M, Xie S -W, Chen Y -L, Xia Y -X and Zhou S -K 1999 Phys. Rev. A 603321 Sun Y H and Li F L 2006 Acta Physica Sinica 551153

[31] Wu S D, Qu Z J, Zhan Z M and Jin L X 2001 Acta Physica Sinica 501925

[32] Bastin T and Martin J 2005 Phys. Rev. A 72053815

[33] Schlicher R 1989 Opt. Commun. 7097 Larson J and Stenholm S 2003 J. Mod. Opt. 501663 Larson J and Stenholm S 2004 J. Mod. Opt. 51129

[34] Larson J and Stenholm S 2003 J. Mod. Opt. 502705

[35] Larson J and Stenholm S 2006 Phys. Rev. A 73033805

[36] Wang D, Larson Å, Karlsson H O, Hansson T and Larson J 2008 Phys. Rev. A 77053808

[37] Griffiths D J 1995 Introduction to Quantum Mechanics (Prentice-Hall) Galindo A and Pascual P 1990 Quantum Mechanics I (Berlin: Springer-Verlag).

[38] Araki H and Lieb E 1970 Commun. Math. Phys. 18160

[39] Heller E J 1975 J. Chem. Phys. 621544

[40] Fleit M D, Fleck J A and Steiger A 1982 J. Comput. Phys. 47412 
[41] Razavy M 2003 Quantum Theory of Tunneling (World Scientific) Ankerhold J 2007 Quantum Tunneling in Complex Systems (Berlin: Springer-Verlag)

[42] Eberly J H, Narozhny N B and Sanchez-Mondragon J J 1980 Phys. Rev. Lett. 441323 Rempe G, Walther H and Klein N 1987 Phys. Rev. Lett. 58353 Larson J 2008 Physica Scr. 76102011

[43] Scully M O and Zubairy M S 1997 Quantum Optics (Berlin: Wiley)

[44] Du S, Zhou L W, Gong S Q, Xu Z Z and Lu J 1999 J. Phys. B: At. Mol. Opt. Phys. 325645

[45] Mandel L and Wolf E 1995 Optical Coherence and Quantum Optics (Cambridge: Cambridge University Press)

[46] Robinett R W 2004 Phys. Rep. 3921

[47] Folman R, Kruger P, Schmiedmayer J, Denschlag J and Henkel C 2002 Adv. At. Mol. Opt. Phys. 48263 Hinds E A, Vale C J and Boshier M G 2001 Phys. Rev. Lett. 861462

Sauer J A, Fortier K M, Chang M S, Hamley C D and Chapman M S 2004 Phys. Rev. A 69051804

[48] Alexanian M and Bose S K 1995 Phys. Rev. A 522218

[49] Ng H T 2008 Phys. Rev. A 77033617 